\title{
Supporting Information for Modeling the Formation and Composition of Secondary Organic Aerosol from Diesel Exhaust Using Parameterized and Semi-explicit Chemistry and Thermodynamic Models
}

Sailaja Eluri ${ }^{1}$, Christopher D. Cappa ${ }^{2}$, Beth Friedman ${ }^{3}$, Delphine K. Farmer ${ }^{3}$, and Shantanu H. Jathar ${ }^{1}$

${ }^{1}$ Department of Mechanical Engineering, Colorado State University, Fort Collins, CO, USA, 80523

${ }^{2}$ Department of Civil and Environmental Engineering, University of California Davis, Davis, CA, USA, 95616

${ }^{3}$ Department of Chemistry, Colorado State University, Fort Collins, CO, USA, 80523

Correspondence to: Shantanu H. Jathar (shantanu.jathar@colostate.edu)

Table S1: Mass speciation and $k_{O H}$ for VOC emissions profile \#3161

\begin{tabular}{|c|c|c|}
\hline Species Name & $k_{O H}\left(\mathrm{~cm}^{3}\right.$ molecules $^{-1} \mathrm{~s}^{-}$ & Mass Percent (\%) \\
\hline (1-methylpropyl) benzene & $8.50 \times 10^{-12}$ & 0.023 \\
\hline (2-methylpropyl) benzene & $8.71 \times 10^{-12}$ & 0.060 \\
\hline 1,2,3-trimethylbenzene & $3.27 \times 10^{-11}$ & 0.056 \\
\hline 1,2,4-trimethylbenzene & $3.25 \times 10^{-11}$ & 0.246 \\
\hline 1,2-diethylbenzene & $8.11 \times 10^{-12}$ & 0.042 \\
\hline 1,2-propadiene & $9.82 \times 10^{-12}$ & 0.218 \\
\hline 1,3,5-trimethylbenzene & $5.67 \times 10^{-11}$ & 0.088 \\
\hline 1,3-butadiene & $6.66 \times 10^{-11}$ & 0.088 \\
\hline 1-butene & $3.14 \times 10^{-11}$ & 0.311 \\
\hline 1-methyl-2-ethylbenzene & $7.44 \times 10^{-12}$ & 0.065 \\
\hline 1-methyl-3-ethylbenzene & $1.39 \times 10^{-11}$ & 0.116 \\
\hline 1-pentene & $3.14 \times 10^{-11}$ & 0.148 \\
\hline 2,2,4-trimethylpentane & $3.34 \times 10^{-12}$ & 0.139 \\
\hline 2,2-dimethylbutane & $2.23 \times 10^{-12}$ & 0.028 \\
\hline 2,3,4-trimethylpentane & $6.60 \times 10^{-12}$ & 0.009 \\
\hline 2,3-dimethyl-1-butene & $5.38 \times 10^{-11}$ & 0.014 \\
\hline 2,3-dimethylhexane & $8.55 \times 10^{-12}$ & 0.005 \\
\hline 2,3-dimethylpentane & $7.14 \times 10^{-12}$ & 0.032 \\
\hline 2,4-dimethylhexane & $8.55 \times 10^{-12}$ & 0.019 \\
\hline 2,4-dimethylpentane & $4.77 \times 10^{-12}$ & 0.009 \\
\hline 2-methylheptane & $8.28 \times 10^{-12}$ & 0.028 \\
\hline 2-methylhexane & $6.86 \times 10^{-12}$ & 0.056 \\
\hline 2-methylpentane & $5.20 \times 10^{-12}$ & 0.181 \\
\hline 3,3-dimethyl-1-butene & $2.80 \times 10^{-11}$ & 1.308 \\
\hline 3-ethylhexane & $8.97 \times 10^{-12}$ & 0.028 \\
\hline 3-methylhexane & $7.15 \times 10^{-12}$ & 0.162 \\
\hline 3-methylpentane & $5.20 \times 10^{-12}$ & 0.056 \\
\hline acetaldehyde & $1.50 \times 10^{-11}$ & 3.409 \\
\hline acetone & $1.70 \times 10^{-13}$ & 3.483 \\
\hline acetylene & $8.15 \times 10^{-13}$ & 1.971 \\
\hline alkene ketone & $1.70 \times 10^{-13}$ & 0.812 \\
\hline b-methylstyrene & $3.12 \times 10^{-11}$ & 0.023 \\
\hline benzaldehyde & $1.20 \times 10^{-11}$ & 0.325 \\
\hline benzene & $1.22 \times 10^{-12}$ & 0.928 \\
\hline butyraldehyde & $2.40 \times 10^{-11}$ & 0.867 \\
\hline c10 aromatics & $2.30 \times 10^{-11}$ & 0.037 \\
\hline c5 aldehyde & $2.88 \times 10^{-11}$ & 0.051 \\
\hline c6 aldehydes & $2.88 \times 10^{-11}$ & 1.763 \\
\hline c9 aromatics & $2.31 \times 10^{-11}$ & 0.232 \\
\hline cis-2-butene & $5.64 \times 10^{-11}$ & 0.042 \\
\hline
\end{tabular}




\begin{tabular}{|c|c|c|}
\hline cis-2-pentene & $6.50 \times 10^{-11}$ & 0.014 \\
\hline cyclohexane & $6.97 \times 10^{-12}$ & 0.014 \\
\hline cyclohexanone & $6.40 \times 10^{-12}$ & 0.051 \\
\hline cyclopentane & $4.97 \times 10^{-12}$ & 0.005 \\
\hline ethane & $2.48 \times 10^{-13}$ & 0.264 \\
\hline ethyl alcohol & $3.58 \times 10^{-12}$ & 0.005 \\
\hline ethylbenzene & $7.00 \times 10^{-12}$ & 0.144 \\
\hline ethylene & $8.52 \times 10^{-12}$ & 6.670 \\
\hline formaldehyde & $9.37 \times 10^{-12}$ & 6.823 \\
\hline indan & $1.90 \times 10^{-11}$ & 0.088 \\
\hline isobutane & $2.44 \times 10^{-12}$ & 0.566 \\
\hline isobutylene & $5.14 \times 10^{-11}$ & 0.427 \\
\hline butylbenzene & $4.50 \times 10^{-12}$ & 0.060 \\
\hline diethylbenzene & $8.11 \times 10^{-12}$ & 0.065 \\
\hline isopentane & $3.60 \times 10^{-12}$ & 0.278 \\
\hline isopropylbenzene & $6.90 \times 10^{-12}$ & 0.009 \\
\hline$m$-xylene & $2.31 \times 10^{-11}$ & 0.283 \\
\hline methane & $6.4 \times 10^{-15}$ & 1.892 \\
\hline methyl alcohol & $6.16 \times 10^{-13}$ & 0.014 \\
\hline methyl ethyl ketone & $1.22 \times 10^{-12}$ & 0.686 \\
\hline 2-hexanone & $9.10 \times 10^{-12}$ & 0.417 \\
\hline methylcyclohexane & $9.64 \times 10^{-12}$ & 0.032 \\
\hline methylcyclopentane & $5.66 \times 10^{-12}$ & 0.070 \\
\hline$n$-butane & $2.36 \times 10^{-12}$ & 0.046 \\
\hline$n$-decane & $1.10 \times 10^{-11}$ & 0.246 \\
\hline$n$-heptane & $6.76 \times 10^{-12}$ & 0.032 \\
\hline$n$-hexane & $5.20 \times 10^{-12}$ & 0.074 \\
\hline$n$-nonane & $9.70 \times 10^{-12}$ & 0.107 \\
\hline$n$-octane & $8.11 \times 10^{-12}$ & 0.065 \\
\hline$n$-pentane & $3.80 \times 10^{-12}$ & 0.083 \\
\hline$n$-propylbenzene & $5.8 \times 10^{-12}$ & 0.056 \\
\hline$n$-undecane & $1.23 \times 10^{-11}$ & 0.121 \\
\hline naphthalene & $2.30 \times 10^{-11}$ & 0.042 \\
\hline$o$-xylene & $1.36 \times 10^{-11}$ & 0.158 \\
\hline$p$-xylene & $1.43 \times 10^{-11}$ & 0.046 \\
\hline propane & $1.09 \times 10^{-12}$ & 0.088 \\
\hline propionaldehyde & $2.20 \times 10^{-11}$ & 0.450 \\
\hline propylene & $2.64 \times 10^{-11}$ & 1.206 \\
\hline styrene & $5.80 \times 10^{-11}$ & 0.028 \\
\hline t-butylbenzene & $4.50 \times 10^{-12}$ & 0.005 \\
\hline unknown & $1.23 \times 10^{-11}$ & 0.093 \\
\hline trans-2-butene & $6.40 \times 10^{-11}$ & 0.019 \\
\hline trans-2-pentene & $6.70 \times 10^{-11}$ & 0.682 \\
\hline toluene & $5.63 \times 10^{-12}$ & 2.268 \\
\hline $\mathrm{C}_{12}$ branched alkane & $1.82 \times 10^{-11}$ & 1.623 \\
\hline $\mathrm{C}_{13}$ branched alkane & $1.68 \times 10^{-11}$ & 1.052 \\
\hline $\mathrm{C}_{14}$ branched alkane & $1.39 \times 10^{-11}$ & 0.939 \\
\hline $\mathrm{C}_{15}$ branched alkane & $1.82 \times 10^{-11}$ & 0.988 \\
\hline $\mathrm{C}_{16}$ branched alkane & $1.96 \times 10^{-11}$ & 0.440 \\
\hline $\mathrm{C}_{17}$ branched alkane & $2.1 \times 10^{-11}$ & 0.573 \\
\hline $\mathrm{C}_{18}$ branched alkane & $2.24 \times 10^{-11}$ & 0.343 \\
\hline $\mathrm{C}_{19}$ branched alkane & $2.38 \times 10^{-11}$ & 0.194 \\
\hline $\mathrm{C}_{20}$ branched alkane & $2.52 \times 10^{-11}$ & 0.128 \\
\hline $\mathrm{C}_{21}$ branched alkane & $2.67 \times 10^{-11}$ & 0.121 \\
\hline $\mathrm{C}_{22}$ branched alkane & $2.81 \times 10^{-11}$ & 8.690 \\
\hline $\mathrm{C}_{12}$ cyclic alkane & $1.82 \times 10^{-11}$ & 8.858 \\
\hline
\end{tabular}




\begin{tabular}{|l|c|c|}
\hline $\mathrm{C}_{13}$ cyclic alkane & $1.68 \times 10^{-11}$ & 6.299 \\
\hline $\mathrm{C}_{14}$ cyclic alkane & $1.39 \times 10^{-11}$ & 5.723 \\
\hline $\mathrm{C}_{15}$ cyclic alkane & $1.82 \times 10^{-11}$ & 4.372 \\
\hline $\mathrm{C}_{16}$ cyclic alkane & $1.96 \times 10^{-11}$ & 3.711 \\
\hline $\mathrm{C}_{17}$ cyclic alkane & $2.1 \times 10^{-11}$ & 3.382 \\
\hline $\mathrm{C}_{18}$ cyclic alkane & $2.24 \times 10^{-11}$ & 2.115 \\
\hline $\mathrm{C}_{19}$ cyclic alkane & $2.38 \times 10^{-11}$ & 1.181 \\
\hline $\mathrm{C}_{20}$ cyclic alkane & $2.52 \times 10^{-11}$ & 0.748 \\
\hline $\mathrm{C}_{21}$ cyclic alkane & $2.67 \times 10^{-11}$ & 0.629 \\
\hline $\mathrm{C}_{22}$ cyclic alkane & $2.81 \times 10^{-11}$ & 1.167 \\
\hline$n$-dodecane & $1.82 \times 10^{-11}$ & 1.094 \\
\hline$n$-tridecane & $1.68 \times 10^{-11}$ & 0.730 \\
\hline$n$-tetradecane & $1.39 \times 10^{-11}$ & 0.613 \\
\hline$n$-pentadecane & $1.82 \times 10^{-11}$ & 0.456 \\
\hline$n$-hexadecane & $1.96 \times 10^{-11}$ & 0.331 \\
\hline$n$-heptadecane & $2.1 \times 10^{-11}$ & 0.296 \\
\hline$n$-octadecane & $2.24 \times 10^{-11}$ & 0.145 \\
\hline$n$-nonadecane & $2.38 \times 10^{-11}$ & 0.073 \\
\hline$n$-eicosane & $2.52 \times 10^{-11}$ & 0.044 \\
\hline$n$-heneicosane & $2.67 \times 10^{-11}$ & 0.029 \\
\hline$n$-docosane & $2.81 \times 10^{-11}$ & 0.287 \\
\hline pristane & $2.44 \times 10^{-11}$ & 0.160 \\
\hline phytane & $2.61 \times 10^{-11}$ & 0.208 \\
\hline naphthalene & $2.3 \times 10^{-11}$ & 0.023 \\
\hline phenanthrene & $1.3 \times 10^{-11}$ & 0.023 \\
\hline
\end{tabular}

Table S2: Mass speciation and $k_{O H}$ for VOC emissions profile \#8774

\begin{tabular}{|l|c|c|}
\hline Species Name & $\boldsymbol{k}_{\text {OH }}\left(\mathbf{c m}^{3}\right.$ molecules $\left.^{-1} \boldsymbol{s}^{-1}\right)$ & Mass Percent (\%) \\
\hline $1,2,3,5$-tetramethylbenzene & $4.30 \times 10^{-10}$ & 0.069 \\
\hline 1,2,3-trimethylbenzene & $3.27 \times 10^{-10}$ & 0.050 \\
\hline $1,2,4,5$-tetramethylbenzene & $2.05 \times 10^{-10}$ & 0.033 \\
\hline 1,2,4-trimethylbenzene & $3.25 \times 10^{-10}$ & 0.021 \\
\hline 1,2 -butadiene & $6.66 \times 10^{-10}$ & 0.010 \\
\hline $1,3,5$-trimethylbenzene & $5.67 \times 10^{-10}$ & 0.042 \\
\hline 1,3-butadiene & $6.66 \times 10^{-10}$ & 0.344 \\
\hline 1,3-diethylbenzene & $1.42 \times 10^{-10}$ & 0.068 \\
\hline 1,3-hexadiene & $1.06 \times 10^{-09}$ & 0.015 \\
\hline 1,4-diethylbenzene & $8.10 \times 10^{-11}$ & 0.091 \\
\hline 1-butene & $3.14 \times 10^{-10}$ & 0.872 \\
\hline 1-heptene & $3.15 \times 10^{-10}$ & 0.152 \\
\hline 1-methyl-2-ethylbenzene & $7.43 \times 10^{-11}$ & 0.078 \\
\hline 1-methyl-3-ethylbenzene & $1.38 \times 10^{-10}$ & 0.047 \\
\hline 1-methyl-4-ethylbenzene & $7.43 \times 10^{-11}$ & 0.039 \\
\hline 1-methylindan & $9.16 \times 10^{-11}$ & 0.028 \\
\hline 1-pentene & $3.14 \times 10^{-10}$ & 0.183 \\
\hline 1-propyne & $7.13 \times 10^{-11}$ & 0.089 \\
\hline 2,2,4-trimethylpentane & $3.34 \times 10^{-11}$ & 0.262 \\
\hline 2,2,5-trimethylhexane & $6.04 \times 10^{-11}$ & 0.057 \\
\hline 2,2-dimethylbutane & $1.89 \times 10^{-11}$ & 0.043 \\
\hline 2,3,4-trimethylpentane & $6.60 \times 10^{-11}$ & 0.032 \\
\hline 2,3-dimethyl-2-pentene & $1.11 \times 10^{-09}$ & 0.002 \\
\hline 2,3-dimethylbutane & $5.44 \times 10^{-11}$ & 0.067 \\
\hline 2,3-dimethylhexane & $8.55 \times 10^{-11}$ & 0.007 \\
\hline 2,3-dimethylpentane & $7.13 \times 10^{-11}$ & 0.078 \\
\hline 2,4-dimethylpentane & $4.77 \times 10^{-11}$ & 0.243 \\
\hline 2,6-dimethylheptane & $9.68 \times 10^{-11}$ & 0.000 \\
\hline
\end{tabular}




\begin{tabular}{|c|c|c|}
\hline 2-methyl-1-butene & $5.26 \times 10^{-10}$ & 0.103 \\
\hline 2-methyl-1-pentene & $5.40 \times 10^{-10}$ & 2.833 \\
\hline 2-methyl-2-butene & $8.73 \times 10^{-10}$ & 0.117 \\
\hline 2-methyl-2-pentene & $8.82 \times 10^{-10}$ & 0.012 \\
\hline 2-methylheptane & $8.27 \times 10^{-11}$ & 0.062 \\
\hline 2-methylhexane & $6.86 \times 10^{-11}$ & 0.070 \\
\hline 2-methylindan & $9.41 \times 10^{-11}$ & 0.017 \\
\hline 2-methylpentane & $5.20 \times 10^{-11}$ & 0.039 \\
\hline 3-methyl-cis-2-pentene & $8.82 \times 10^{-10}$ & 0.009 \\
\hline 3-methyl-trans-2-pentene & $8.82 \times 10^{-10}$ & 0.111 \\
\hline 3-methylheptane & $8.56 \times 10^{-11}$ & 0.054 \\
\hline 3-methylhexane & $7.14 \times 10^{-11}$ & 0.130 \\
\hline 3-methyloctane & $9.97 \times 10^{-11}$ & 0.142 \\
\hline 4-methylheptane & $8.27 \times 10^{-11}$ & 0.018 \\
\hline acetaldehyde & $1.50 \times 10^{-10}$ & 2.228 \\
\hline acetylene & $8.15 \times 10^{-12}$ & 2.675 \\
\hline alpha-pinene & $9.07 \times 10^{-10}$ & 0.035 \\
\hline benzaldehyde & $1.20 \times 10^{-10}$ & 0.231 \\
\hline benzene & $1.22 \times 10^{-11}$ & 1.281 \\
\hline beta-pinene & $5.65 \times 10^{-10}$ & 0.001 \\
\hline butyraldehyde & $2.40 \times 10^{-10}$ & 0.514 \\
\hline cis-2-butene & $5.64 \times 10^{-10}$ & 0.098 \\
\hline cis-2-hexene & $5.90 \times 10^{-10}$ & 0.021 \\
\hline cis-2-pentene & $6.50 \times 10^{-10}$ & 0.053 \\
\hline crotonaldehyde & $3.61 \times 10^{-10}$ & 0.128 \\
\hline cyclohexane & $6.97 \times 10^{-11}$ & 0.120 \\
\hline cyclohexene & $6.15 \times 10^{-10}$ & 0.051 \\
\hline cyclopentane & $4.97 \times 10^{-11}$ & 0.038 \\
\hline cyclopentene & $5.87 \times 10^{-10}$ & 0.060 \\
\hline dl-limonene & $1.45 \times 10^{-09}$ & 0.012 \\
\hline ethane & $2.48 \times 10^{-12}$ & 0.739 \\
\hline ethylbenzene & $7.00 \times 10^{-11}$ & 0.147 \\
\hline ethylene & $8.52 \times 10^{-11}$ & 8.180 \\
\hline formaldehyde & $9.37 \times 10^{-11}$ & 3.554 \\
\hline glyoxal & $2.53 \times 10^{-10}$ & 0.296 \\
\hline indan & $8.27 \times 10^{-11}$ & 0.028 \\
\hline isobutane & $2.44 \times 10^{-11}$ & 0.305 \\
\hline isopentane & $3.60 \times 10^{-11}$ & 1.721 \\
\hline isoprene & $1.05 \times 10^{-09}$ & 0.061 \\
\hline isopropylbenzene & $6.90 \times 10^{-11}$ & 0.013 \\
\hline isopropylcyclohexane & $1.33 \times 10^{-10}$ & 0.150 \\
\hline isopropyltoluene & $8.53 \times 10^{-11}$ & 0.062 \\
\hline$m \& p$-xylene & $2.31 \times 10^{-10}$ & 0.252 \\
\hline 2-butanone & $1.22 \times 10^{-11}$ & 1.198 \\
\hline methylcyclohexane & $9.64 \times 10^{-11}$ & 0.115 \\
\hline methylcyclopentane & $5.66 \times 10^{-11}$ & 1.786 \\
\hline$n$-butane & $2.36 \times 10^{-11}$ & 0.573 \\
\hline$n$-butylbenzene & $8.72 \times 10^{-11}$ & 0.021 \\
\hline$n$-decane & $1.10 \times 10^{-10}$ & 0.404 \\
\hline$n$-heptane & $6.76 \times 10^{-11}$ & 0.125 \\
\hline$n$-hexane & $5.20 \times 10^{-11}$ & 0.307 \\
\hline$n$-nonane & $9.70 \times 10^{-11}$ & 0.159 \\
\hline$n$-octane & $8.11 \times 10^{-11}$ & 0.145 \\
\hline$n$-pentane & $3.80 \times 10^{-11}$ & 0.313 \\
\hline$n$-propylbenzene & $5.80 \times 10^{-11}$ & 0.026 \\
\hline$n$-undecane & $1.23 \times 10^{-10}$ & 0.417 \\
\hline
\end{tabular}




\begin{tabular}{|c|c|c|}
\hline$O$-xylene & $1.36 \times 10^{-10}$ & 0.104 \\
\hline$n$-propane & $1.09 \times 10^{-11}$ & 0.890 \\
\hline propionaldehyde & $2.19 \times 10^{-10}$ & 0.257 \\
\hline propylene & $2.64 \times 10^{-10}$ & 2.347 \\
\hline propyltoluene & $8.80 \times 10^{-11}$ & 0.050 \\
\hline styrene & $5.80 \times 10^{-10}$ & 0.129 \\
\hline tolualdehyde & $1.86 \times 10^{-10}$ & 0.016 \\
\hline toluene & $5.63 \times 10^{-12}$ & 0.588 \\
\hline trans-1,3-dichloropropene & $9.35 \times 10^{-11}$ & 0.002 \\
\hline trans-2-butene & $6.40 \times 10^{-10}$ & 0.121 \\
\hline trans-2-hexene & $6.66 \times 10^{-10}$ & 0.036 \\
\hline trans-2-pentene & $6.7 \times 10^{-10}$ & 0.054 \\
\hline valeraldehyde & $2.74 \times 10^{-10}$ & 0.023 \\
\hline $\mathrm{C}_{12}$ branched alkane & $1.82 \times 10^{-11}$ & 1.623 \\
\hline $\mathrm{C}_{13}$ branched alkane & $1.68 \times 10^{-11}$ & 1.052 \\
\hline $\mathrm{C}_{14}$ branched alkane & $1.39 \times 10^{-11}$ & 0.939 \\
\hline $\mathrm{C}_{15}$ branched alkane & $1.82 \times 10^{-11}$ & 0.988 \\
\hline $\mathrm{C}_{16}$ branched alkane & $1.96 \times 10^{-11}$ & 0.440 \\
\hline $\mathrm{C}_{17}$ branched alkane & $2.1 \times 10^{-11}$ & 0.573 \\
\hline $\mathrm{C}_{18}$ branched alkane & $2.24 \times 10^{-11}$ & 0.343 \\
\hline $\mathrm{C}_{19}$ branched alkane & $2.38 \times 10^{-11}$ & 0.194 \\
\hline $\mathrm{C}_{20}$ branched alkane & $2.52 \times 10^{-11}$ & 0.128 \\
\hline $\mathrm{C}_{21}$ branched alkane & $2.67 \times 10^{-11}$ & 0.121 \\
\hline $\mathrm{C}_{22}$ branched alkane & $2.81 \times 10^{-11}$ & 8.690 \\
\hline $\mathrm{C}_{12}$ cyclic alkane & $1.82 \times 10^{-11}$ & 8.858 \\
\hline $\mathrm{C}_{13}$ cyclic alkane & $1.68 \times 10^{-11}$ & 6.299 \\
\hline $\mathrm{C}_{14}$ cyclic alkane & $1.39 \times 10^{-11}$ & 5.723 \\
\hline $\mathrm{C}_{15}$ cyclic alkane & $1.82 \times 10^{-11}$ & 4.372 \\
\hline $\mathrm{C}_{16}$ cyclic alkane & $1.96 \times 10^{-11}$ & 3.711 \\
\hline $\mathrm{C}_{17}$ cyclic alkane & $2.1 \times 10^{-11}$ & 3.382 \\
\hline $\mathrm{C}_{18}$ cyclic alkane & $2.24 \times 10^{-11}$ & 2.115 \\
\hline $\mathrm{C}_{19}$ cyclic alkane & $2.38 \times 10^{-11}$ & 1.181 \\
\hline $\mathrm{C}_{20}$ cyclic alkane & $2.52 \times 10^{-11}$ & 0.748 \\
\hline $\mathrm{C}_{21}$ cyclic alkane & $2.67 \times 10^{-11}$ & 0.629 \\
\hline $\mathrm{C}_{22}$ cyclic alkane & $2.81 \times 10^{-11}$ & 1.167 \\
\hline$n$-dodecane & $1.82 \times 10^{-11}$ & 1.094 \\
\hline$n$-tridecane & $1.68 \times 10^{-11}$ & 0.730 \\
\hline$n$-tetradecane & $1.39 \times 10^{-11}$ & 0.613 \\
\hline n-pentadecane & $1.82 \times 10^{-11}$ & 0.456 \\
\hline$n$-hexadecane & $1.96 \times 10^{-11}$ & 0.331 \\
\hline$n$-heptadecane & $2.1 \times 10^{-11}$ & 0.296 \\
\hline$n$-octadecane & $2.24 \times 10^{-11}$ & 0.145 \\
\hline$n$-nonadecane & $2.38 \times 10^{-11}$ & 0.073 \\
\hline$n$-eicosane & $2.52 \times 10^{-11}$ & 0.044 \\
\hline$n$-heneicosane & $2.67 \times 10^{-11}$ & 0.029 \\
\hline$n$-docosane & $2.81 \times 10^{-11}$ & 0.287 \\
\hline pristane & $2.44 \times 10^{-11}$ & 0.160 \\
\hline phytane & $2.61 \times 10^{-11}$ & 0.208 \\
\hline naphthalene & $2.30 \times 10^{-11}$ & 0.023 \\
\hline phenanthrene & $1.30 \times 10^{-11}$ & 0.023 \\
\hline
\end{tabular}

Table S3: Mass speciation and $k_{O H}$ for VOC emissions profile \#4777

\begin{tabular}{|l|c|c|}
\hline Species Name & $\boldsymbol{k}_{\mathrm{OH}}\left(\mathbf{c m}^{3}\right.$ molecules $\left.^{-1} \mathbf{s}^{-1}\right)$ & Mass Percent (\%) \\
\hline 1,1,4-trimethylcyclohexane & $9.09 \times 10^{-12}$ & 0.081 \\
\hline 1,2,4-trimethylbenzene & $1.67 \times 10^{-11}$ & 0.405 \\
\hline 1,2-dimethyl-4-ethylbenzene & $1.69 \times 10^{-11}$ & 0.177 \\
\hline
\end{tabular}




\begin{tabular}{|c|c|c|}
\hline 1,3,5-trimethylbenzene & $3.51 \times 10^{-11}$ & 0.162 \\
\hline 1,3-dimethyl-2-ethylbenzene & $1.76 \times 10^{-11}$ & 0.283 \\
\hline 1,4-dimethyl-2-ethylbenzene & $1.69 \times 10^{-11}$ & 0.374 \\
\hline 1-(1,1-dimethylethyl)-3,5-dimethylbenzene & $3.01 \times 10^{-11}$ & 0.319 \\
\hline 1-butene & $3.14 \times 10^{-11}$ & 0.521 \\
\hline 1-ethyl-1-methylcyclopentane & $6.33 \times 10^{-12}$ & 0.035 \\
\hline 1-hexene & $3.02 \times 10^{-11}$ & 0.152 \\
\hline 1-methyl-2-ethylbenzene & $7.44 \times 10^{-12}$ & 0.329 \\
\hline 1-methyl-2-tert-butylbenzene & $6.74 \times 10^{-12}$ & 0.369 \\
\hline 1-methyl-3-ethylbenzene & $1.39 \times 10^{-11}$ & 0.617 \\
\hline 1-methyl-3-isopropylbenzene & $1.45 \times 10^{-11}$ & 0.379 \\
\hline 1-methyl-3-propylbenzene & $1.52 \times 10^{-11}$ & 0.233 \\
\hline 1-methyl-4-ethylbenzene & $7.44 \times 10^{-12}$ & 0.182 \\
\hline 1-nonene & $3.44 \times 10^{-11}$ & 0.061 \\
\hline 1-pentene & $3.14 \times 10^{-11}$ & 0.273 \\
\hline 1-tert-butyl-4-ethylbenzene & $7.42 \times 10^{-12}$ & 0.167 \\
\hline 2,2,4-trimethylpentane & $3.16 \times 10^{-11}$ & 0.197 \\
\hline 2,2-dimethylbutane & $1.82 \times 10^{-12}$ & 0.101 \\
\hline 2,2-dimethylpropane & $6.69 \times 10^{-13}$ & 0.051 \\
\hline 2,3,4-trimethylpentane & $8.54 \times 10^{-12}$ & 0.046 \\
\hline 2,3-dimethylbutane & $5.44 \times 10^{-12}$ & 0.020 \\
\hline 2,3-dimethylhexane & $5.09 \times 10^{-12}$ & 0.106 \\
\hline 2,3-dimethylpentane & $7.14 \times 10^{-12}$ & 0.015 \\
\hline 2,4-dimethylhexane & $4.92 \times 10^{-12}$ & 0.051 \\
\hline 2,4-dimethylpentane & $6.85 \times 10^{-12}$ & 0.010 \\
\hline 2,5-dimethylheptane & $9.97 \times 10^{-12}$ & 0.071 \\
\hline 2,5-dimethylhexane & $7.24 \times 10^{-12}$ & 0.025 \\
\hline 2-methyl-butyl-benzene & $1.02 \times 10^{-11}$ & 0.946 \\
\hline 2-methylhexane & $6.86 \times 10^{-12}$ & 0.293 \\
\hline 2-methylnonane & $1.11 \times 10^{-11}$ & 0.273 \\
\hline 2-methyloctane & $9.97 \times 10^{-12}$ & 0.091 \\
\hline 2-methylpentane & $5.45 \times 10^{-12}$ & 0.040 \\
\hline 3,3-dimethyloctane & $7.21 \times 10^{-12}$ & 0.263 \\
\hline 3,3-dimethylpentane & $2.97 \times 10^{-12}$ & 0.081 \\
\hline 3,5-dimethylheptane & $1.02 \times 10^{-11}$ & 0.076 \\
\hline 3-ethyloctane & $1.18 \times 10^{-11}$ & 0.162 \\
\hline 3-ethylpentane & $7.56 \times 10^{-12}$ & 0.030 \\
\hline 3-methyl-1-butene & $2.86 \times 10^{-11}$ & 0.111 \\
\hline 3-methyl-cis-2-pentene & $8.83 \times 10^{-11}$ & 0.071 \\
\hline 3-methylheptane & $8.56 \times 10^{-12}$ & 0.056 \\
\hline 3-methylhexane & $7.15 \times 10^{-12}$ & 0.020 \\
\hline 3-methylnonane & $1.14 \times 10^{-11}$ & 0.228 \\
\hline 3-methylpentane & $5.73 \times 10^{-12}$ & 0.071 \\
\hline 4-methyl-1-pentene & $3.02 \times 10^{-11}$ & 0.061 \\
\hline 4-methylheptane & $1.02 \times 10^{-11}$ & 0.475 \\
\hline acetaldehyde & $1.50 \times 10^{-11}$ & 2.710 \\
\hline acetone & $1.70 \times 10^{-13}$ & 3.828 \\
\hline acetylene & $8.15 \times 10^{-13}$ & 2.346 \\
\hline acrolein & $2.58 \times 10^{-11}$ & 0.759 \\
\hline benzene & $1.22 \times 10^{-12}$ & 1.370 \\
\hline cis,trans-1,2,4-trimethylcyclohexane & $1.35 \times 10^{-11}$ & 3.580 \\
\hline cis-2-butene & $5.64 \times 10^{-11}$ & 0.056 \\
\hline cis-2-nonene & $6.32 \times 10^{-11}$ & 0.096 \\
\hline cis-2-octene & $6.18 \times 10^{-11}$ & 0.046 \\
\hline cis-3-hexene & $2.00 \times 10^{-10}$ & 0.066 \\
\hline crotonaldehyde & $3.62 \times 10^{-11}$ & 0.228 \\
\hline
\end{tabular}




\begin{tabular}{|c|c|c|}
\hline cyclohexane & $6.97 \times 10^{-12}$ & 0.015 \\
\hline cyclohexane & $6.97 \times 10^{-12}$ & 0.071 \\
\hline cyclopentane & $4.97 \times 10^{-12}$ & 0.030 \\
\hline cyclopentene & $5.88 \times 10^{-11}$ & 0.046 \\
\hline ethane & $2.48 \times 10^{-13}$ & 0.172 \\
\hline ethylbenzene & $7.00 \times 10^{-12}$ & 0.071 \\
\hline ethylene & $8.52 \times 10^{-12}$ & 10.013 \\
\hline formaldehyde & $9.37 \times 10^{-12}$ & 9.330 \\
\hline isobutane & $2.44 \times 10^{-12}$ & 0.030 \\
\hline isopentane & $3.60 \times 10^{-12}$ & 0.344 \\
\hline isopropylcyclohexane & $1.34 \times 10^{-11}$ & 0.126 \\
\hline isopropyltoluene & $8.54 \times 10^{-12}$ & 0.308 \\
\hline$m \& p$-xylene & $2.31 \times 10^{-11}$ & 0.319 \\
\hline 2-butanone & $1.22 \times 10^{-12}$ & 1.643 \\
\hline methylbutadiene & $1.05 \times 10^{-10}$ & 0.091 \\
\hline methylcyclohexane & $5.09 \times 10^{-12}$ & 0.086 \\
\hline methylcyclopentane & $5.66 \times 10^{-12}$ & 0.030 \\
\hline$n$-butane & $2.36 \times 10^{-12}$ & 0.081 \\
\hline$n$-butylcyclopentane & $1.01 \times 10^{-11}$ & 0.137 \\
\hline$n$-decane & $1.11 \times 10^{-11}$ & 1.461 \\
\hline$n$-dodecane & $1.39 \times 10^{-11}$ & 1.193 \\
\hline$n$-heptane & $6.76 \times 10^{-12}$ & 0.040 \\
\hline$n$-hexane & $5.20 \times 10^{-12}$ & 0.152 \\
\hline$n$-nonane & $9.70 \times 10^{-12}$ & 0.738 \\
\hline$n$-octane & $8.11 \times 10^{-12}$ & 0.415 \\
\hline$n$-pentane & $3.80 \times 10^{-12}$ & 0.076 \\
\hline$n$-pentylbenzene & $1.01 \times 10^{-11}$ & 0.182 \\
\hline$n$-propylbenzene & $5.80 \times 10^{-12}$ & 0.137 \\
\hline$n$-tridecane & $1.53 \times 10^{-11}$ & 0.526 \\
\hline$n$-undecane & $1.23 \times 10^{-11}$ & 1.664 \\
\hline$O$-xylene & $1.36 \times 10^{-11}$ & 0.339 \\
\hline$n$-propane & $1.09 \times 10^{-12}$ & 0.025 \\
\hline propionaldehyde & $2.20 \times 10^{-11}$ & 0.541 \\
\hline propylcyclopentane & $1.20 \times 10^{-11}$ & 0.051 \\
\hline propylene & $2.64 \times 10^{-11}$ & 2.195 \\
\hline toluene & $5.63 \times 10^{-12}$ & 1.406 \\
\hline trans-1,2-dimethylcyclopentane & $6.80 \times 10^{-12}$ & 1.310 \\
\hline trans-1,4-dimethylcyclohexane & $1.19 \times 10^{-11}$ & 0.056 \\
\hline trans-2-butene & $6.40 \times 10^{-11}$ & 0.076 \\
\hline trans-2-heptene & $6.80 \times 10^{-11}$ & 0.030 \\
\hline trans-2-octene & $6.94 \times 10^{-11}$ & 0.071 \\
\hline trans-2-pentene & $6.70 \times 10^{-11}$ & 0.046 \\
\hline trans-3-nonene & $7.04 \times 10^{-11}$ & 0.000 \\
\hline $\mathrm{C}_{12}$ branched alkane & $1.82 \times 10^{-11}$ & 1.623 \\
\hline $\mathrm{C}_{13}$ branched alkane & $1.68 \times 10^{-11}$ & 1.052 \\
\hline $\mathrm{C}_{14}$ branched alkane & $1.39 \times 10^{-11}$ & 0.939 \\
\hline $\mathrm{C}_{15}$ branched alkane & $1.82 \times 10^{-11}$ & 0.988 \\
\hline $\mathrm{C}_{16}$ branched alkane & $1.96 \times 10^{-11}$ & 0.440 \\
\hline $\mathrm{C}_{17}$ branched alkane & $2.1 \times 10^{-11}$ & 0.573 \\
\hline $\mathrm{C}_{18}$ branched alkane & $2.24 \times 10^{-11}$ & 0.343 \\
\hline $\mathrm{C}_{19}$ branched alkane & $2.38 \times 10^{-11}$ & 0.194 \\
\hline $\mathrm{C}_{20}$ branched alkane & $2.52 \times 10^{-11}$ & 0.128 \\
\hline $\mathrm{C}_{21}$ branched alkane & $2.67 \times 10^{-11}$ & 0.121 \\
\hline $\mathrm{C}_{22}$ branched alkane & $2.81 \times 10^{-11}$ & 8.690 \\
\hline $\mathrm{C}_{12}$ cyclic alkane & $1.82 \times 10^{-11}$ & 8.858 \\
\hline $\mathrm{C}_{13}$ cyclic alkane & $1.68 \times 10^{-11}$ & 6.299 \\
\hline
\end{tabular}




\begin{tabular}{|l|c|c|}
\hline $\mathrm{C}_{14}$ cyclic alkane & $1.39 \times 10^{-11}$ & 5.723 \\
\hline $\mathrm{C}_{15}$ cyclic alkane & $1.82 \times 10^{-11}$ & 4.372 \\
\hline $\mathrm{C}_{16}$ cyclic alkane & $1.96 \times 10^{-11}$ & 3.711 \\
\hline $\mathrm{C}_{17}$ cyclic alkane & $2.1 \times 10^{-11}$ & 3.382 \\
\hline $\mathrm{C}_{18}$ cyclic alkane & $2.24 \times 10^{-11}$ & 2.115 \\
\hline $\mathrm{C}_{19}$ cyclic alkane & $2.38 \times 10^{-11}$ & 1.181 \\
\hline $\mathrm{C}_{20}$ cyclic alkane & $2.52 \times 10^{-11}$ & 0.748 \\
\hline $\mathrm{C}_{21}$ cyclic alkane & $2.67 \times 10^{-11}$ & 0.629 \\
\hline $\mathrm{C}_{22}$ cyclic alkane & $2.81 \times 10^{-11}$ & 1.167 \\
\hline$n$-dodecane & $1.82 \times 10^{-11}$ & 1.094 \\
\hline$n$-tridecane & $1.68 \times 10^{-11}$ & 0.730 \\
\hline$n$-tetradecane & $1.39 \times 10^{-11}$ & 0.613 \\
\hline$n$-pentadecane & $1.82 \times 10^{-11}$ & 0.456 \\
\hline$n$-hexadecane & $1.96 \times 10^{-11}$ & 0.331 \\
\hline$n$-heptadecane & $2.1 \times 10^{-11}$ & 0.296 \\
\hline$n$-octadecane & $2.24 \times 10^{-11}$ & 0.145 \\
\hline$n$-nonadecane & $2.38 \times 10^{-11}$ & 0.073 \\
\hline$n$-eicosane & $2.52 \times 10^{-11}$ & 0.044 \\
\hline$n$-heneicosane & $2.67 \times 10^{-11}$ & 0.029 \\
\hline$n$-docosane & $2.81 \times 10^{-11}$ & 0.287 \\
\hline pristane & $2.44 \times 10^{-11}$ & 0.160 \\
\hline phytane & $2.61 \times 10^{-11}$ & 0.208 \\
\hline naphthalene & $2.3 \times 10^{-11}$ & 0.023 \\
\hline Phenanthrene & $1.3 \times 10^{-11}$ & 0.023 \\
\hline
\end{tabular}

Table S4:VBS and SOM model performance for OA mass at $0 \%, 13.76 \%$, and $60 \%$ IVOC mass fractions

\begin{tabular}{|c|c|c|c|c|c|c|c|c|c|c|}
\hline \multirow{2}{*}{ Aftertreatment } & \multirow{2}{*}{ Model } & \multicolumn{2}{|c|}{ Fractional Bias } & \multicolumn{2}{|c|}{ Fractional Error } & \multicolumn{3}{|c|}{$\boldsymbol{R}^{2}$} \\
\cline { 3 - 11 } & & $\mathbf{0 \%}$ & $\mathbf{1 3 . 6 \%}$ & $\mathbf{6 0 \%}$ & $\mathbf{0 \%}$ & $\mathbf{1 3 . 6 \%}$ & $\mathbf{6 0 \%}$ & $\mathbf{0 \%}$ & $\mathbf{1 3 . 6 \%}$ & $\mathbf{6 0 \%}$ \\
\hline \multirow{2}{*}{ None } & VBS & $-128 \%$ & $-13 \%$ & $77 \%$ & $136 \%$ & $84 \%$ & $91 \%$ & 0.83 & 0.92 & 0.9 \\
\cline { 2 - 11 } & SOM & $-125 \%$ & $-63 \%$ & $70 \%$ & $138 \%$ & $108 \%$ & $90 \%$ & 0.83 & 0.94 & 0.92 \\
\hline \multirow{2}{*}{ DPF+DOC } & VBS & $-148 \%$ & $-121 \%$ & $-46 \%$ & $161 \%$ & $133 \%$ & $99 \%$ & 0.77 & 096 & 0.99 \\
\cline { 2 - 11 } & SOM & $-145 \%$ & $-82 \%$ & $28 \%$ & $169 \%$ & $128 \%$ & $65 \%$ & 0.52 & 0.95 & 0.99 \\
\hline \multirow{2}{*}{ All } & VBS & $-134 \%$ & $-49 \%$ & $36 \%$ & $144 \%$ & $100 \%$ & $82 \%$ & 0.81 & 0.92 & 0.92 \\
\cline { 2 - 10 } & SOM & $-135 \%$ & $-70 \%$ & $56 \%$ & $149 \%$ & $115 \%$ & $93 \%$ & 0.77 & 0.93 & 0.93 \\
\hline
\end{tabular}

Table S5: SOM performance for O:C predictions at 0\%,13.76\%, and $60 \%$ IVOC mass fractions.

\begin{tabular}{|c|c|c|c|c|c|c|c|c|c|}
\hline \multirow{2}{*}{ Aftertreatment } & \multicolumn{3}{|c|}{ Fractional Bias } & \multicolumn{2}{|c|}{ Fractional Eror } & \multicolumn{3}{|c|}{$\boldsymbol{R}^{2}$} \\
\cline { 2 - 10 } & $\mathbf{0 \%}$ & $\mathbf{1 3 . 6 \%}$ & $\mathbf{6 0 \%}$ & $\mathbf{0 \%}$ & $\mathbf{1 3 . 6 \%}$ & $\mathbf{6 0 \%}$ & $\mathbf{0 \%}$ & $\mathbf{1 3 . 6 \%}$ & $\mathbf{6 0 \%}$ \\
\hline None & $-18 \%$ & $-46 \%$ & $-63 \%$ & $26 \%$ & $46 \%$ & $65 \%$ & 0.86 & 0.85 & 0.69 \\
\hline DPF+DOC & $29 \%$ & $-31 \%$ & $-84 \%$ & $35 \%$ & $36 \%$ & $84 \%$ & 0.38 & 0.02 & 0.29 \\
\hline All & $-3 \%$ & $-41 \%$ & $-55 \%$ & $29 \%$ & $43 \%$ & $57 \%$ & 0.80 & 0.75 & 0.49 \\
\hline
\end{tabular}




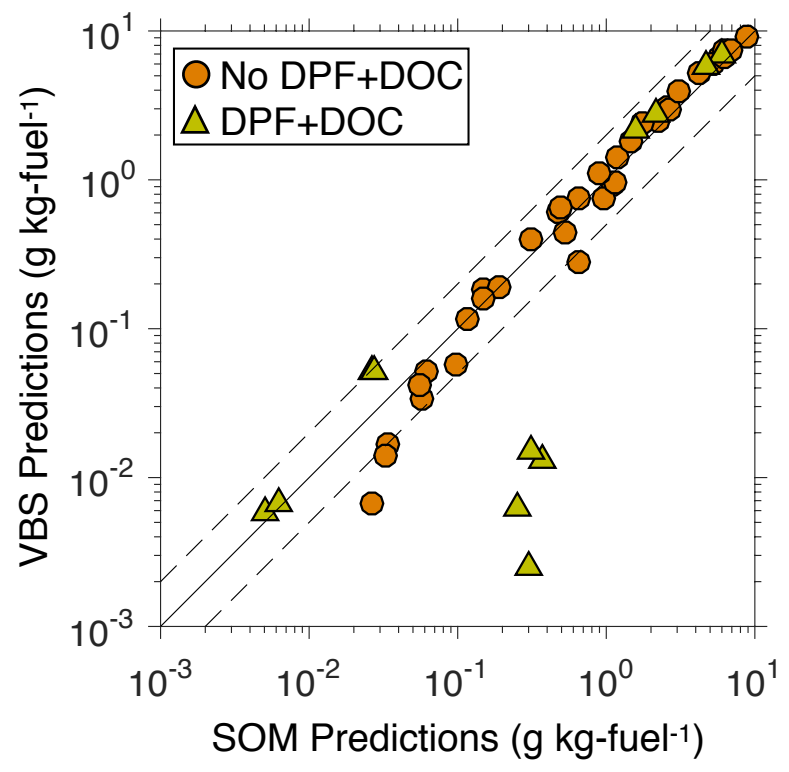

Figure S1: Scatter plot comparing SOM predictions of OA mass to VBS predictions from all experiments at all photochemical ages for the base case.

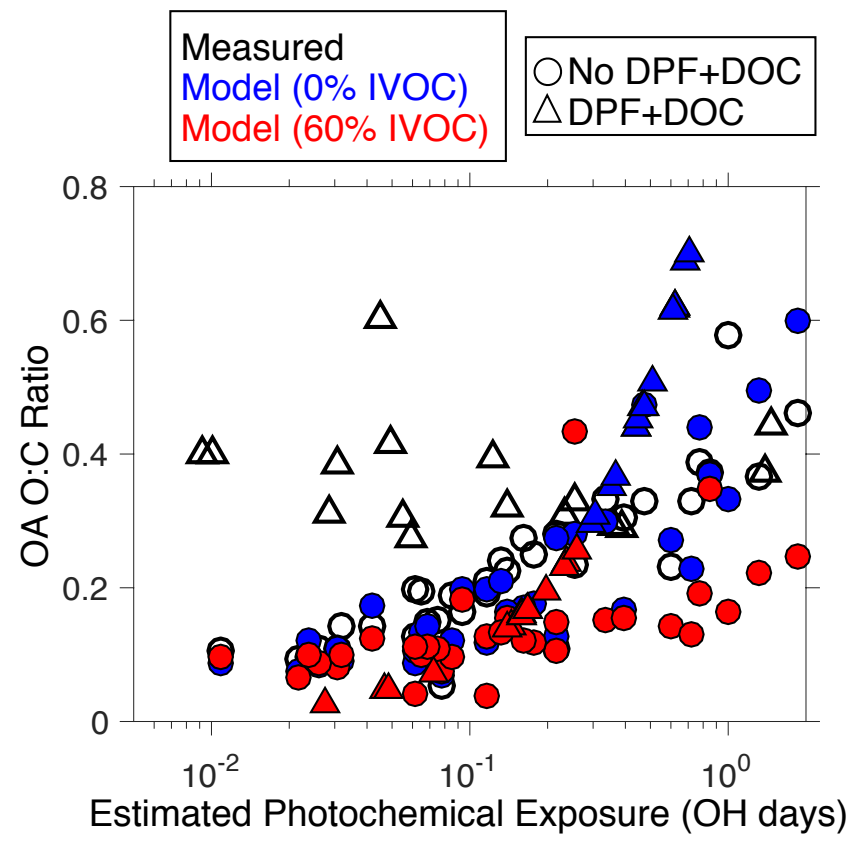

Figure S2: Measurements and model predictions from the 0\% and 60\% IVOC case of the OA O:C ratio presented as a function of the photochemical age. 